Research Article

\title{
Modification of Gravitational Field Equation due to Invariance of Light Speed and New System of Universe Evolution
}

\author{
Jian Liang Yang (i) \\ College of Physics, Zhengzhou University, Zhengzhou 450001, China \\ Correspondence should be addressed to Jian Liang Yang; bps267890@qq.com \\ Received 16 February 2021; Revised 4 March 2021; Accepted 9 March 2021; Published 20 March 2021 \\ Academic Editor: Ghulam Abbas \\ Copyright (C) 2021 Jian Liang Yang. This is an open access article distributed under the Creative Commons Attribution License, \\ which permits unrestricted use, distribution, and reproduction in any medium, provided the original work is properly cited.

\begin{abstract}
We make a systematic examination of the basic theory of general relativity and reemphasize the meaning of coordinates. Firstly, we prove that Einstein's gravitational field equation has the light speed invariant solution and black holes are not an inevitable prediction of general relativity. Second, we show that the coupling coefficient of the gravitational field equation is not unique and can be modified as $4 \pi G$ to replace the previous $-8 \pi G$, distinguish gravitational mass from the inertial mass, and prove that dark matter and dark energy are not certain existence and the expansion and contraction of the universe are proven cyclic, and a new distance-redshift relation which is more practical is derived. After that, we show that galaxies and celestial bodies are formed by gradual growth rather than by the accumulation of existing matter and prove that new matter is generating gradually in the interior of celestial bodies. For example, the radius of the Earth increases by $0.5 \mathrm{~mm}$ every year, and its mass increases by 1.2 trillion tons. A more reasonable derivation of the precession of planetary orbits is given, and the evolution equation of planetary orbits in the expanding space-time is also given. In a word, an alive universe unfolds in front of readers and the current cosmological difficulties are given new interpretations.
\end{abstract}

\section{Introduction}

Although general relativity has made some remarkable achievements, some basic problems have not been well solved, such as the physical meaning of the coordinates of Schwarzschild metric, whether general relativity is the curved theory of space-time or the theory of gravity in flat space-time, whether the constant speed of light is also tenable in the gravitational field, the singularity problem of the field equation, and whether the existence of black holes is true. However, only these basic problems have plagued the development of general relativity but also led to some confusion in practice; for example, on the one hand, the radial coordinates of Schwarzschild metric are not interpreted as the normal radius, while, on the other hand, the radial coordinates on the solar surface are treated as the radius of the sun in calculating the curvature of light on the surface of the sun, resulting in conceptual confusion. In addition, there are some new observations that are not accommodated by the current gravity theory. As Lorio [1] pointed out, there is an unexplained increase in the distance between the Sun and the Earth, and after considering the tides, the moon still has an unexplained retreat, and the increase of the day length is also inconsistent with the prediction of the tide theory. Recently, Melissa Ness and her colleagues have observed that there is a fine X-shaped box structure in vortex galaxies similar to the Milky Way [2]. Melissa Ness said that this structure implies that large galaxies are not formed by the merger of small galaxies, because once the merger occurs, the structure will inevitably be destroyed, and we must abandon the existing theory of galaxy formation and establish a new logic system. The observations of Martinez-Lombila and others [3] show that the radius of disk galaxies similar to the Milky Way galaxy is expanding at a speed of $500 \mathrm{~m} / \mathrm{s}$; such a high speed cannot be the speed at which matter accumulates at the edge. If matter accumulates at this speed at the edge, it should be the same everywhere on the disk. Obviously, the current theoretical framework cannot explain such a rapid expansion of the radius of the disk. There is also the problem of dark matter and dark energy; the reason why we need them is that the observed phenomena do not conform to the prediction of the theory, but, no one has seen them really. Then, whether they are real or the theory itself needs to be modified is also 
an unavoidable problem. The latest observation data of Nielsen and others [4] show that the universe is expanding at a constant speed rather than accelerating, so whether the universe accelerates or decelerates or expands at constant speed still needs to be reconsidered. Besides, some new studies of frontier disciplines $[5,6]$ have shown that 1 billion years ago, the brightness of the sun was less than half of what it is today, the Earth is an ice ball, and the mountain is not as high as it is today, and 2.7 billion years ago, the air pressure on the Earth was only half of today's. These seem to be purely geophysical problems, which can only be reasonably explained from the perspective of cosmology because the evolution of the Earth is an epitome of the evolution of the universe and the Earth must be reflected by cosmological events. On the contrary, the phenomena on the Earth can be used to test the cosmological theory more accurately and people do not have to go far to test the theory of cosmology. In a word, we are faced with some new problems that cannot be avoided. We will see that when the speed limit of light, that is, the speed of light always 1 (in natural units), is still satisfied in the gravitational field, the above problems can be solved in a package. The author thinks that it is a great mistake of general relativity that the invariance of the speed of light in the gravitational field is not emphasized in the past, and it is this fault that leads to a series of misconceptions and absurd results; for example, it is necessary to admit singularity as physical reality, which will never be allowed in other parts of physics. In a word, it is shameless to tie the correctness of general relativity with some wrong conclusions such as big bang and black holes, and it is shameless to praise mistakes as successes. Leading to the big bang, black holes and all kinds of other singularities are not the success of general relativity, but its failure. The reason is simple: there is no singularity in real nature. No matter how much you boast big bang and black holes, they cannot be true. The author thinks that if these absurd things are not stripped away from general relativity, there will be no real progress in general relativity, the field of astrophysics will be dominated by all kinds of idealism, and more and more young students will be misled into the wrong way. In order to deal with these problems systematically, to get to the bottom and bring order out from chaos, this paper begins with the most basic problem, that is, solving the metric of the spherically symmetric gravitational field represented by coordinates in the usual sense.

\section{Spherically Symmetric Static Metric Represented in Usual Coordinates}

We just have to solve for the metric form in the usual spherical coordinates; the form in other coordinates can be obtained by coordinate transformation. Indices $\mu, \nu, \lambda, \alpha, \beta=0,1,2,3$. Space-time coordinates $x^{\mu}=\left(x^{0}, x^{1}\right.$, $\left.x^{2}, x^{3}\right)=(t, r, \theta, \varphi)$ and $x^{0}=t, x^{1}=r, x^{2}=\theta, x^{3}=\varphi$ represent the usual time, radius, and pole angles, respectively. They have the same meaning as in quantum mechanics or electrodynamics. In the language of the observational theory of general relativity, $t$ is the time recorded by a stationary observer at infinite distance, $r$ is the distance the observer measures from the origin to another point, and $\theta, \varphi$ are the polar angles measured by the observer.
In this paper, we use natural units, the speed of light of flat space-time $c=1$, and it is agreed that flat space-time linear element is

$$
\mathrm{d} s^{2}=g_{\mu \nu} \mathrm{d} x^{\mu} \mathrm{d} x^{\nu}=\mathrm{d} t^{2}-\mathrm{d} r^{2}-r^{2}\left(\mathrm{~d} \theta^{2}+\sin ^{2} \theta \mathrm{d} \varphi^{2}\right) .
$$

According to general relativity, in a spherically symmetric gravitational field, in the coordinate system $(t, r, \theta, \varphi)$, the general form of space-time line elements is [7-11]

$$
\begin{aligned}
\mathrm{d} s^{2}= & g_{\mu \nu} \mathrm{d} x^{\mu} \mathrm{d} x^{\nu}=B(r, t) \mathrm{d} t^{2}-Q(r, t) \mathrm{d} t \mathrm{~d} r \\
& -A(r, t) \mathrm{d} r^{2}-D(r, t)\left(\mathrm{d} \theta^{2}+\sin ^{2} \theta \mathrm{d} \varphi^{2}\right) .
\end{aligned}
$$

The condition of this formula is only spherical symmetry, which is applicable to the gravitational field of both static and oscillating gravitational sources. In this paper, we will just deal with the static gravitational field, which is what Newtonian gravity describes. For the static case, $g_{\mu \nu}$ no longer contains time. Besides, the static case requires time version to be symmetric, so $g_{01}=Q(r)=0$. Therefore, for the static case of spherical symmetry, the space-time line element is

$$
\mathrm{d} s^{2}=g_{\mu \nu} \mathrm{d} x^{\mu} \mathrm{d} x^{\nu}=B(r) \mathrm{d} t^{2}-A(r) \mathrm{d} r^{2}-D(r)\left(\mathrm{d} \theta^{2}+\sin ^{2} \theta \mathrm{d} \varphi^{2}\right) .
$$

We just have to solve for three functions $B(r), A(r)$, and $D(r)$. In order to ensure that the meaning of coordinates is always clear and unchanged, this paper will not continue to simplify (3) into the so-called standard form through coordinate transformation but directly solve with the gravitational field equation. Firstly, determine the external solution that satisfies the vacuum field equation $R_{\mu \nu}=0$, and then the source internal solution is determined.

In order to reflect the invariance of light speed, we require $A(r)=B(r)$. From the following solving process, we can see that such a solution not only exists but also is unique. Equation (3) provides

$$
\begin{aligned}
& g_{00}=B(r), \\
& g_{11}=-A(r), \\
& g_{\mu \nu}=0(\mu \neq \nu), \\
& g_{22}=-D(r), \\
& g_{33}=-D(r) \sin ^{2} \theta, \\
& g^{22}=-\frac{1}{D(r)}, \\
& g^{33}=-\frac{1}{D(r) \sin ^{2} \theta}, \\
& g^{00}=\frac{1}{B(r)}, \\
& g^{11}=-\frac{1}{A(r)}, \\
& g^{\mu \nu}=0(\mu \neq \nu) .
\end{aligned}
$$


According to the definition of connection, $\Gamma_{\mu \nu}^{\lambda}=(1 / 2) g^{\lambda \alpha}\left(\partial g_{\alpha \mu} / \partial x^{\nu}+t \partial g_{\alpha \nu} / \partial x^{\mu} n-q \partial g_{\mu \nu} / \partial x^{\alpha}\right)$, the repeating indices up and down means summing from 0 to 3 , and it is not hard to figure out all of its nonzero connections as follows [2-7]:

$$
\begin{aligned}
& \Gamma_{33}^{1}=-\frac{1}{2 A} \frac{\partial D}{\partial r} \sin ^{2} \theta, \\
& \Gamma_{23}^{3}=\frac{\cos \theta}{\sin \theta}, \\
& \Gamma_{11}^{1}=\frac{1}{2} \frac{\partial A}{\partial r}, \\
& \Gamma_{01}^{0}=\frac{1}{2 B} \frac{\partial B}{\partial r}, \\
& \Gamma_{00}^{1}=\frac{1}{2 A} \frac{\partial B}{\partial r}, \\
& \Gamma_{12}^{2}=\Gamma_{13}^{3} \\
& \Gamma_{22}^{1}=-\frac{1}{2 A} \frac{\partial D}{\partial r}, \\
& \Gamma_{33}^{2}=-\sin \theta \cos \theta .
\end{aligned}
$$

According to the definition of curvature tensor, $R_{\mu \nu}=\partial \Gamma_{\mu \lambda}^{\lambda} / \partial x^{\nu}-\partial \Gamma_{\mu \nu}^{\lambda} / \partial x^{\lambda}+\Gamma_{\mu \lambda}^{\alpha} \Gamma_{\alpha \nu}^{\lambda}-\Gamma_{\mu \nu}^{\alpha} \Gamma_{\alpha \lambda}^{\lambda}$; for $\mu \neq \nu$, we have $R_{\mu \nu}=0$, which means that the vacuum field equation is automatically satisfied. It is not hard to figure out all of the nonzero components of $R_{\mu v}$. Write $A^{\prime}=\mathrm{d} A / \mathrm{d} r$, $A^{\prime \prime}=\mathrm{d}^{2} A / \mathrm{d} r^{2}, \quad A^{\prime 2}=(\mathrm{d} A / \mathrm{d} r)^{2}, \quad$ and note that $R_{33}=R_{22} \sin ^{2} \theta$; we are left with the following three equations about $B(r), A(r)$, and $D(r)$ :

$$
\begin{aligned}
& R_{00}=-\left(\frac{B^{\prime}}{2 A}\right)^{\prime}+\frac{B^{\prime 2}}{2 A B}-\frac{B^{\prime}}{2 A}\left(\frac{A^{\prime}}{2 A}+\frac{D^{\prime}}{D}+\frac{B^{\prime}}{2 B}\right)=0 \\
& R_{11}=\left(\frac{D^{\prime}}{D}+\frac{B^{\prime}}{2 B}\right)^{\prime}+\left(\frac{D^{\prime 2}}{2 D^{2}}+\frac{B^{\prime 2}}{4 B^{2}}\right)-\frac{A^{\prime}}{2 A}\left(\frac{D^{\prime}}{D}+\frac{B^{\prime}}{2 B}\right)=0 \\
& R_{22}=\frac{D^{\prime \prime}}{2 A}+\frac{D^{\prime}}{4 A}\left(-\frac{A^{\prime}}{A}+\frac{B^{\prime}}{B}\right)-1=0 . \\
& \text { From } R_{00} \times(1 / B)+R_{11} \times(1 / A)=0 \text { we obtain } \\
& -\frac{A B^{\prime}+A^{\prime} B}{2 A B}\left(\frac{D^{\prime}}{D}\right)+\left(\frac{D^{\prime}}{D}\right)^{\prime}+\frac{D^{\prime 2}}{2 D^{2}}=-\frac{(A B)^{\prime}}{2 A B} \frac{D^{\prime}}{D}+\left(\frac{D^{\prime}}{D}\right)^{\prime} \\
& +\frac{D^{\prime 2}}{2 D^{2}}=0 .
\end{aligned}
$$

Equation (9) is a differential equation with respect to $A B$, its general solution is $A B=C_{1} D^{\prime 2} / D$, and $C_{1}$ is the integral constant. Since $A=B=1, D=r^{2}, D^{\prime}=2 r, D^{\prime \prime}=2$ at infinity, there must be $C_{1}=1 / 4$, namely, $A B=D^{\prime 2} / 4 D$. And inserting $A=D^{\prime 2} / 4 B D$ into equation (8) gets

$$
B^{\prime}+\frac{D^{\prime}}{2 D} B-\frac{D^{\prime}}{2 D}=0
$$

which is a differential equation with respect to $B$. Writing $D=l^{2}$, the general solution of (10) is given by $B=1+C_{2} / l$. $C_{2}$ is an integral constant. Because we must return to Newton gravitation in the distance, we have $C_{2}=-2 G M$. $G$ Newton's gravitational constant $M$ is the mass of the source.

It is important to insert $B=1-2 G M / l$ and $A=D^{\prime 2} / 4 B D=l^{\prime} /(1-2 G M / l)$ into any one of (6)-(8). You can obtain an identity with respect to $l=l(r)$; namely, no matter what $l=l(r)$ is, this identity can be tenable, so we can pick an appropriate $l(r)$ so that $A=B$. And letting $A=B$, namely, $1-2 G M / l=\mathrm{d} l / \mathrm{d} r$, we obtain

$$
r=C_{3}+l+2 G M \ln (l-2 G M),
$$

where $C_{3}$ is the integral constant and can be decided by the continuity of $l(r)$ on the surface of the source. In Section 4 of this paper, $C_{3}$ will be calculated. So far, we obtain the exterior line element:

$$
\mathrm{d} s^{2}=\left(1-\frac{2 G M}{l}\right) \mathrm{d} t^{2}-\left(1-\frac{2 G M}{l}\right) \mathrm{d} r^{2}-l^{2}\left(\mathrm{~d} \theta^{2}+\sin ^{2} \theta \mathrm{d} \varphi^{2}\right),
$$

where $l=l(r)$ can be inversely solved from (11). It can be seen from (11) that when $l=2 G M, r$ becomes negative, which means $l-2 G M$ is always greater than 0 and so there is no horizon and no black hole. And considering $\lim _{x \longrightarrow \infty}(\ln x / x)=0$, we have $r=l$ for $l \longrightarrow \infty$.

\section{Link with the Mechanics of Special Relativity}

The invariance of the speed of light described by (11) is easy to see. Suppose that the photon moves in the radial direction, $\mathrm{d} \varphi=\mathrm{d} \theta=0, \quad \mathrm{~d} s^{2}=0, \quad$ and from (11), we can get $\mathrm{d} r^{2} / \mathrm{d} t^{2}=B(r) / A(r)=1$, namely, $\mathrm{d} r / \mathrm{d} t= \pm 1$, which shows that radial speed of light is constant. And now we look at the light moving tangentially and set $\theta=\pi / 2, \mathrm{~d} r=0, \mathrm{~d} s^{2}=0$; the tangential speed of light is given from (12) by $r \mathrm{~d} \varphi / \mathrm{d} t=(r / l) \sqrt{1-2 G M / l}$. And (11) tells us that the smaller $\sqrt{1-2 G M / l}$ than 1 , the larger $r / l$ than 1 , so the deviation of $(r / l) \sqrt{1-2 G M / l}$ from 1 is actually very small, and you can still think of it as 1 . It is in this sense that we say that (11) describes the invariant speed of light, not strictly constant. This slight change of the tangential speed of light causes light to bend near a celestial body; otherwise, it travels in straight lines. The previous result is $r \mathrm{~d} \varphi / \mathrm{d} t=\sqrt{1-2 G M / r}$ which can be obtained from (28), and it is not hard to find that when $r=2 G M$, the tangential speed of light is zero and the deviation from 1 is severe; although it means also that light can bend, it does not reflect the invariance of the speed of light.

The correctness of (12) is not only in the invariance of the speed of light but also in the natural connection with relativistic mechanics under weak field approximation. Equation (12) provides 


$$
\begin{aligned}
& \Gamma_{01}^{1}=0, \\
& \Gamma_{11}^{1}=\Gamma_{01}^{0}=\frac{G M}{(1-2 G M / l) l^{2}} \frac{\mathrm{d} l}{\mathrm{~d} r}=\frac{G M}{l^{2}}, \\
& \Gamma_{00}^{1}=\frac{G M}{l^{2}} .
\end{aligned}
$$

The dynamic equation describing the motion of free particles is the geodesic equation, the proper time must be eliminated when solving for acceleration, and the geodesic equation after the elimination of proper time is

$$
\frac{\mathrm{d}^{2} x^{\mu}}{\mathrm{d} t^{2}}+\Gamma_{\nu \lambda}^{\mu} \frac{\mathrm{d} x^{\nu}}{\mathrm{d} t} \cdot \frac{\mathrm{d} x^{\lambda}}{\mathrm{d} t}-\Gamma_{\nu \lambda}^{0} \frac{\mathrm{d} x^{\nu}}{\mathrm{d} t} \cdot \frac{\mathrm{d} x^{\lambda}}{\mathrm{d} t} \cdot \frac{\mathrm{d} x^{\mu}}{\mathrm{d} t}=0,
$$

which is derived in detail in post-Newtonian mechanics [9]. Let the particle move on the plan $\theta=\pi / 2$, set $\mu=1,3$, and write $\mathrm{d} r / \mathrm{d} t=v_{r}, \mathrm{~d} \varphi / \mathrm{d} t=v_{\varphi} / r$; we have

$$
\frac{\mathrm{d}^{2} r}{\mathrm{~d} t^{2}}=-\Gamma_{00}^{1}-\Gamma_{11}^{1} \dot{r}^{2}+2 \Gamma_{01}^{0} \dot{r}^{2}+\Gamma_{33}^{1} \dot{\varphi}^{2}=-\frac{G M}{l^{2}}+\frac{G M v_{r}^{2}}{l^{2}}+\frac{l v_{\varphi}^{2}}{r^{2}},
$$

$\frac{\mathrm{d}^{2} \varphi}{\mathrm{d} t^{2}}=-2 \Gamma_{13}^{3} \dot{r} \dot{\varphi}+2 \Gamma_{01}^{0} \dot{r} \dot{\varphi}=-\frac{2 l^{\prime}}{r l} v_{r} v_{\varphi}+\frac{1}{1-2 G M / l} \frac{2 G M}{l^{2} r} v_{r} v_{\varphi}$.

In the weak field or in the distance, $2 G M / l \ll 1, l \longrightarrow r$, $l^{2} \longrightarrow r^{2}, l^{\prime} \longrightarrow 1,(15)$ and (16) become, respectively,

$$
\begin{aligned}
& \frac{\mathrm{d}^{2} r}{\mathrm{~d} t^{2}}+\frac{G M}{r^{2}}-\frac{G M v_{r}^{2}}{r^{2}}-\frac{v_{\varphi}^{2}}{r}=0, \\
& \frac{\mathrm{d}^{2} \varphi}{\mathrm{d} t^{2}}+\frac{2}{r^{2}} v_{r} v_{\varphi}-\frac{2 G M}{r^{3}} v_{r} v_{\varphi}=0 .
\end{aligned}
$$

Ignoring the higher-order small quantity $2 G M / r^{3},(17)$ becomes

$$
\frac{\mathrm{d}^{2} \varphi}{\mathrm{d} t^{2}}+\frac{2}{r^{2}} v_{r} v_{\varphi}=0
$$

It is not difficult to prove that (17) and (19) are just the relativistic Newton equations of gravity:

$$
\frac{\mathrm{d}(m \mathbf{v})}{\mathrm{d} t}=-\frac{G M m}{r^{2}} \mathbf{e}_{r}
$$

where $m=m_{0} / \sqrt{1-v^{2}}$ is the motion mass of the particle, $\mathbf{v}=\dot{r} \mathbf{e}_{r}+r \dot{\varphi} \mathbf{e}_{\varphi}, v^{2}=\dot{r}^{2}+r^{2} \dot{\varphi}^{2}$, and $\mathbf{e}_{r}, \mathbf{e}_{\varphi}$ are the base vectors. Prove as follows: from theoretical mechanics, we know

$$
\begin{aligned}
\frac{\mathrm{d} \mathbf{v}}{\mathrm{d} t} & =\left(\ddot{r}-r \dot{\varphi}^{2}\right) \mathbf{e}_{r}+(2 \dot{r} \dot{\varphi}+r \ddot{\varphi}) \mathbf{e}_{\varphi}, \\
\frac{\mathrm{d} v^{2}}{\mathrm{~d} t} & =2 \dot{r} \ddot{r}+2 r^{2} \dot{\varphi} \ddot{\varphi}+2 r \dot{r} \dot{\varphi}^{2}, \\
\frac{\mathrm{d}(m \mathbf{v})}{\mathrm{d} t} & =m \frac{\mathrm{d} \mathbf{v}}{\mathrm{d} t}+\mathbf{v} \frac{\mathrm{d} m}{\mathrm{~d} t}=m \frac{\mathrm{d} \mathbf{v}}{\mathrm{d} t}+m v \frac{\mathrm{d} v^{2}}{2\left(1-v^{2}\right) \mathrm{d} t}
\end{aligned}
$$

Using (20), we have

$$
\begin{gathered}
\ddot{r} \frac{1-r^{2} \dot{\varphi}^{2}}{1-v^{2}}+\frac{r^{2} \dot{r} \dot{\varphi} \ddot{\varphi}}{1-v^{2}}+\frac{\dot{\varphi}^{2} r \dot{r}^{2}}{1-v^{2}}-r \dot{\varphi}^{2}=-\frac{G M}{r^{2}}, \\
\ddot{\varphi} \frac{r-r \dot{r}^{2}}{1-v^{2}}+\frac{r \dot{r} \dot{\varphi} \ddot{r}}{1-v^{2}}+\frac{r^{2} \dot{\varphi}^{3} \dot{r}}{1-v^{2}}+2 \dot{r} \dot{\varphi}=0,
\end{gathered}
$$

eliminating $\ddot{\varphi}$ in the use $(22) \times\left(1-\dot{r}^{2}\right)-(23) \times r \dot{\varphi} \dot{r}$ gets

$$
\ddot{r}-\frac{v_{\varphi}^{2}}{r}+\frac{G M}{r^{2}}-\frac{G M}{r^{2}} v_{r}^{2}=0,
$$

which is exactly equation (17), and inserting it into (23), we get

$$
\ddot{\varphi}+\frac{2}{r^{2}} v_{r} v_{\varphi}-\frac{G M}{r^{3}} v_{r} v_{\varphi}=0,
$$

which is (19) omitting the higher-order small GMv $v_{r} v_{\varphi} / r^{3}$. So far, it can be seen that (12) in the weak field approximation can link well with the special relativistic mechanics.

And again, when the particle moves along the radial direction, if $v^{2}=1$, the acceleration of the particle is equal to zero, which means that the invariance of light speed and the light speed limit are unified.

\section{The Light Speed Invariant Solution within a Spherically Symmetric Static Gravity Source}

Now, we solve the interior $A(r), B(r)$, and $D(r)$. In order to keep the constant speed of light, we still require $A(r)=B(r)$ inside the source. From the following solution's process, we know such a solution not only exists but also is unique. Note that the constant speed of light means that the speed of light passing through the cavity in the source is 1 , but that passing through the medium is 1 . The equation of gravitational field in the source is

$$
R_{\mu \nu}=\gamma\left(T_{\mu \nu}-\frac{1}{2} T g_{\mu \nu}\right)
$$

where $\gamma$ is the coupling constant, and that we do not write its specific value here is to lay a hint for the following 
modification of the constant. And $T_{\mu \nu}=(\rho+p) u_{\mu} u_{\nu}-p g_{\mu \nu}$ is the energy-momentum tensor of the source. Note that $u^{\mu}=\mathrm{d} x^{\mu} / \mathrm{d} s, u_{\mu}=g_{\mu \nu} u^{\nu}, u_{\mu} u^{\mu}=1, g_{\mu \nu} g^{\mu \nu}=4, T=g^{\mu \nu} T_{\mu \nu}=$ $\rho-3 p$.

And for the static source, $u^{i}=\mathrm{d} x^{i} / \mathrm{d} s=0, i=1,2,3$. Then, $\quad u_{i}=g_{i v} u^{v}=0, \quad 1=B(\mathrm{~d} t / \mathrm{d} s)^{2}=B\left(u^{0}\right)^{2}$, $u_{0}=g_{00} u^{0}=\sqrt{B}, \quad T_{11}=p A, T_{22}=p D, T_{33}=p D \sin ^{2} \theta$, $T_{00}=\rho B$; from (26), we have

$$
R_{00}=-\left(\frac{B^{\prime}}{2 A}\right)^{\prime}+\frac{B^{\prime 2}}{2 A B}-\frac{B^{\prime}}{2 A}\left(\frac{A^{\prime}}{2 A}+\frac{D^{\prime}}{D}+\frac{B^{\prime}}{2 B}\right)=\frac{\gamma}{2}(\rho+3 p) B,
$$

$$
\begin{aligned}
R_{11} & =\left(\frac{D^{\prime}}{D}+\frac{B^{\prime}}{2 B}\right)^{\prime}+\left(\frac{D^{\prime 2}}{2 D^{2}}+\frac{B^{\prime 2}}{4 B^{2}}\right)-\frac{A^{\prime}}{2 A}\left(\frac{D^{\prime}}{D}+\frac{B^{\prime}}{2 B}\right) \\
& =\frac{\gamma}{2}(\rho-p) A,
\end{aligned}
$$

$R_{22}=\frac{D^{\prime \prime}}{2 A}+\frac{D^{\prime}}{4 A}\left(-\frac{A^{\prime}}{A}+\frac{B^{\prime}}{B}\right)-1=\frac{\gamma}{2}(\rho-p) D$.

In the use of $\left(R_{00} / 2 B\right)+\left(R_{11} / 2 A\right)+\left(R_{22} / D\right)=\rho \gamma$, we get

$$
\left(\frac{1}{A}\right)^{\prime}+\left(\frac{2 D^{\prime \prime}}{D^{\prime}}-\frac{D^{\prime}}{2 D}\right) \frac{1}{A}-\frac{2}{D^{\prime}}-2 \gamma \rho \frac{D}{D^{\prime}}=0,
$$

which can be looked as a differential equation with respect to $1 / A$. And writing $D=l^{2}$, in the requirement of ensuring $D=$ 0 and $A$ being limited at origin, the solution of (31) is given by

$$
A=\left(1+\frac{\gamma}{l} \int_{0}^{l} \rho l^{2} \mathrm{~d} l\right)^{-1} l^{2}
$$

where $l^{\prime 2}=(\mathrm{d} l / \mathrm{d} r)^{2}$. Writing $A_{1}=\left(1+l^{-1} \gamma \int_{0}^{l} \rho l^{2} \mathrm{~d} l\right)^{-1}$, we have

$$
\begin{aligned}
A^{\prime} & =A_{1}^{\prime} l^{2}+2 A_{1} l^{\prime} l^{\prime \prime}, \\
\left(l^{2}\right)^{\prime \prime} & =2 l^{\prime 2}+2 l l^{\prime \prime}, \\
B^{\prime} & =\frac{l^{\prime} \mathrm{d} B}{\mathrm{~d} l} .
\end{aligned}
$$

Insert these into (30), we obtain

$$
\frac{\mathrm{d} B}{B \mathrm{~d} l}=-\gamma A_{1}\left(p l+l^{-2} \int_{0}^{l} l^{2} \rho \mathrm{d} l\right) .
$$

To make $B(r)$ continuous on the boundary of source, the solution of (33) is

$$
B=\left(1-\frac{2 G M}{l_{2}}\right) \exp \int_{l_{e}}^{l}-\gamma A_{1}\left(p l+l^{-2} \int_{0}^{l} l^{2} \rho \mathrm{d} l\right) \mathrm{d} l,
$$

where $l_{e}=l\left(r_{e}\right)$ is the value on the boundary and $r_{e}$ is the radius of the source.

It is important that, similar to the external solution, substituting (32) and (34) into any of (28)-(30), you can get the identity with respect to $l=l(r)$; that is, this is true regardless of the function of $l(r)$, regardless of the value of $\gamma$. So, we can pick a function $l(r)$ by the following equation (41) to make $A(r)=B(r)$ :

$$
\begin{aligned}
(1 & \left.-\frac{2 G M}{l_{e}}\right) \exp \int_{l_{e}}^{l}-\gamma A_{1}\left(p l+l^{-2} \int_{0}^{l} l^{2} \rho \mathrm{d} l\right) \mathrm{d} l \\
= & \left(1+l^{-1} \gamma \int_{0}^{l} \rho l^{2} \mathrm{~d} l\right)^{-1} l^{\prime 2} .
\end{aligned}
$$

On the other hand, we know $T_{\mu ; \nu}^{\nu}=0$, for the static source; it is [2-7]

$$
\frac{\partial p}{\partial x^{\mu}}=-(\rho+p) \frac{\partial}{\partial x^{\mu}} \ln \sqrt{B}
$$

\section{Modification of the Coupling Constant and the Internal $D(r)$}

When the pressure at the surface of the gravitational source is set as zero, it can be seen from (39) that once the geodesic equation is required to return to Newtonian gravity under the weak field approximation, the coupling constant $\gamma$ must be $-8 \pi G$, which is the previous result. But this result should be considered as a mistake because it leads to a lot of singularities that should not occur. For example, when the ratio of the mass to the radius of an object is $2 G M / R>8 / 9$, the pressure inside the object becomes infinite [7-9], which is obviously absurd [10-16]. The root of all kinds of singularity is the improper selection of $-8 \pi G$. As we will see, when the pressure is taken negative, the coupling constant is identified as $4 \pi G$, which not only avoid singularity of Schwarzschild metric but also remove in a package cosmological problems.

I need to say a few words about the negative pressure. Einstein did not refuse the negative pressure. In the book The Meaning of Relativity, Princeton University Press Published, 1922, (page 117), for $T_{\mu \nu}=\rho u_{\mu} u_{\nu}$, Einstein said, we "shall add a pressure term that may be physically established as follows. Matter consists of electrically charged particles. On the basis of Maxwell's theory these cannot be conceived of as electromagnetic fields free from singularities. In order to be consistent with the facts, it is necessary to introduce energy terms, not contained in Maxwell's theory, so that the single electric particles may hold together in spite of the mutual repulsion between their elements, charged with electricity of one sign. For the sake of consistency with this fact, Poincare has assumed a pressure to exist inside these particles which balances the electrostatic repulsion. It cannot, however, be asserted that this pressure vanishes outside the particles. We shall be consistent with this circumstance if, in our phenomenological presentation, we add a pressure term. This must not, however, be confused with a hydrodynamical pressure, as it serves only for the energetic presentation of the dynamical relations inside matter." From this statement, it can be seen that Einstein did not equate pressure as a source of gravity with the dynamic pressure of a fluid but regarded it as a phenomenological representation of all the 
action within matter, including the electromagnetic force. It is not surprising that a negative value is taken.

Now solve for $D(r)$ in the source with negative pressure and in the meantime determine the coupling constant. For the convenience of calculation, we use the average density instead of the density of each point that is to take the interior $\rho=$ const. The density itself is a statistical average, such treatment is equivalent to treating the whole celestial body as a statistical volume element, so it is suitable for some not too large celestial bodies as such the sun, and it is also an approximation for larger celestial bodies.

When $\rho$ is regarded as a constant, $p=-\rho$ is the solution of (36). Since the geodesic under the weak field approximation must return to Newtonian gravity, there must be $\Gamma_{00}^{1} \longrightarrow G M / r$ on the surface of the source, and we conclude that, from (33), the coupling constant $\gamma=4 \pi G$. Notice that under the weak field approximation $l \longrightarrow r, A_{1} \longrightarrow 1$, $4 \pi \int_{0}^{l_{e}} \rho l^{2} \mathrm{~d} l \longrightarrow M$, and $p=-\rho \longrightarrow-3 M / 4 \pi r_{e}^{3}$.

With $p=-\rho, \gamma=4 \pi G$, and $l_{e}$ as the fixed value, the integral of both sides of (35) is easy, and we get

$$
\frac{\mathrm{d} l}{\mathrm{~d} r}=\sqrt{\frac{1-2 G M / l_{e}}{1+4 \pi G \rho l_{e}^{2} / 3}}\left(1+\frac{4 \pi G \rho l^{2}}{3}\right) .
$$

Ensuring $B^{\prime}=0$ for $r=0$, which is because the force on the particle at the origin is zero, the solution of (37) is

$$
\sqrt{\frac{3}{4 \pi G \rho}} \operatorname{arctg}\left(l \sqrt{\frac{4 \pi G \rho}{3}}\right)=r \sqrt{\frac{1-2 G M / l_{e}}{1+4 \pi G \rho l_{e}^{2} / 3}} .
$$

On the other hand, we require $\Gamma_{00}^{1}$ to be also continuous on the boundary, which is a necessary condition to ensure the continuity of gravity on the boundary. So, there exists on the boundary

$$
\Gamma_{00}^{1}=\frac{\partial B}{B \partial l} \frac{\partial l}{\partial r}=\frac{8 \pi G \rho l_{e}}{3} \sqrt{\frac{1-2 G M / l_{e}}{1+4 \pi G \rho l_{e}^{2} / 3}}=\Gamma_{00}^{1}=\frac{2 G M}{l_{e}^{2}} .
$$

Solve (39), $\quad M=\sqrt{G^{2} k^{2}+k l_{e}}-G k$, in which $k=16 \pi^{2} \rho^{2} l_{e}^{5} /\left(9+12 \pi G \rho l_{e}^{2}\right)$.

The explicit form of $M$ can be solved under the weak field approximation, and expanding the square root in (39) by Taylor, we obtain

$$
M=\frac{4 \pi \rho l_{e}^{3}}{3}-\frac{8}{3} \pi^{2} \rho^{2} G l_{e}^{5}+\cdots
$$

Applying (39) on the boundary and taking the approximation $M=4 \pi \rho l_{e}^{3} / 3$ into, we have

$$
\sqrt{\frac{3}{4 \pi G \rho}} \operatorname{arctg}\left(l_{e} \sqrt{\frac{4 \pi G \rho}{3}}\right)=r_{e} \sqrt{\frac{1-8 \pi G \rho l_{e}^{2} / 3}{1+4 \pi G \rho l_{e}^{2} / 3}}
$$

Obviously, for $1-8 \pi G \rho l_{e}^{2} / 3=0, r_{e} \longrightarrow \infty$; that is to say, no matter how big the radius of the celestial body, there is always $l_{e}<3 / 8 \pi G \rho$; we do not have to worry about whether $l_{e}$ has a solution. And expanding the two sides of (41) by Taylor and taking second-order approximation, we obtain

$$
\begin{aligned}
& l_{e}=r_{e}-\frac{14}{9} \pi G \rho r_{e}^{3} \\
& r_{e}=l_{e}+\frac{14}{9} \pi G \rho l_{e}^{3}, \\
& l_{e}^{3}=r_{e}^{3}-\frac{14}{3} \pi G \rho r_{e}^{5}
\end{aligned}
$$

Inserting (42) into (40), we obtain

$$
M=\frac{4 \pi \rho}{3} r_{e}^{3}-\frac{80 \pi^{2} \rho^{2} G}{9} r_{e}^{5}+\cdots
$$

Writing $4 \pi \rho r_{e}^{3} / 3=M_{i}$, we have

$$
\begin{aligned}
M & =M_{i}-\frac{5 G}{r_{e}} M_{i}^{2}+\cdots, \\
M_{i} & =M+\frac{5 G}{r_{e}} M^{2}+\cdots,
\end{aligned}
$$

where $M_{i}$ is the inertial mass and $M$ is the gravitational mass. The reason why $M_{i}$ is called inertial mass is that $\rho$ represents the inertial density of matter measured in comoving coordinate system, while $M$ is introduced from the perspective of gravity, so it is natural to call it gravitational mass.

Equation (44) distinguishes gravitational mass from inertial mass, which is the result of an in-depth discussion in this paper, and for high-density celestial bodies, the difference is obvious.

We see $l_{e}=r_{e}-7 G M / 6+\cdots$, and so far, the integral constant $C_{3}$ in (11) can be decided according to the continuity on the boundary; that is (11) is applied to the surface of the source

$$
\begin{aligned}
C_{3}= & r_{e}-l_{e}-2 G M \ln \left(l_{e}-2 G M\right)=r_{e}-l_{e} \\
& -2 G M \ln \left(r_{e}-2 G M\right) \approx \frac{7 G M}{6}-2 G M \ln r_{e} .
\end{aligned}
$$

Inserting $C_{3}$ into (11), we can complete the calculation of Mercury precession and ray bending, and the calculated result is that the difference between the new results and the original ones is very small and completely consistent with the observation. The concrete calculation is not written here, readers can do it by themselves.

In a word, with the new coupling constant $4 \pi G$, the gravitational field equation is now modified as

$$
R_{\mu \nu}=4 \pi G\left(T_{\mu \nu}-\frac{1}{2} T g_{\mu \nu}\right)
$$

and correspondingly the pressure $p$ as the source of gravitation takes negative. Multiplying the two sides of (46) with $g^{\mu \nu}$, we have $R=-4 \pi G T$, so the equivalent form of (46) is $R_{\mu \nu}-(1 / 2) R g_{\mu \nu}=4 \pi G T_{\mu \nu}$.

\section{Further Interpretation of the Physical Meaning of the Negative Pressure}

Einstein did not interpret the pressure term in a gravitational source as the dynamic pressure of a fluid, but as a 
phenomenological representation of the pressure within a matter to balance the electromagnetic force and prevent charged particles from being disintegrated by electrical repulsion, which we should accept. Einstein did not point out that pressure is produced by which power. Today, it is easy to infer and prevent the disintegration of the protons and neutrons are strong; preventing electronics is the disintegration of the weak force. Therefore, the pressure term should be understood as a phenomenological representation of the combined effects of the strong, weak, electromagnetic, gravitational, and all other forms of action within a matter, representing the total binding energy that holds the matter together, represented by the potential energy of the system. In other words, if you divide the matter infinitely, and you move each part to infinity, the work done is the volume integral of the pressure, which is negative, and the absolute value is equal to the mass of the gravitational source, namely, $\int p \mathrm{~d} x \mathrm{~d} y \mathrm{~d} z=-M$, which is like adding a physical condition to make the solution of the pressure definite. In fact, the form of the gravitational source already determines the interpretation of $p$. As gravitational source $T_{\mu \nu}=(\rho+p) u_{\mu} u_{\nu}-p g_{\mu \nu}$, if $p$ is still understood as the common dynamic pressure, then the field equation can only be used to solve for the metric of an ideal fluid, which is obviously not hoped by general relativity, and the dynamic pressure in a solid is generally considered to be zero. let alone (36). Can $p$ be understood as a thermal pressure? No, because the thermal motion is absorbed by $\rho$ in the form of thermal kinetic energy and cannot be repeated to appear. $p$ is called pressure only because it appears in the equation of motion in the form of pressure; of course, $p$ includes the effect of common pressure. The equation of motion refers to $T_{i \nu}^{\mu \nu}=0$.

When the field equation with the coupling constant $4 \pi G$ is applied to the universe, $T_{\mu \nu}=(\rho+p) u_{\mu} u_{\nu}-p g_{\mu \nu}$ represents the energy-momentum tensor of space of the universe, and taking the statistical average of $\rho$ and $p$, we have $p=-\rho$, which is just the equation of state of the dark energy said usually. So, we say that dark energy is just the binding energy of matter, rather than an independent existence.

\section{The Problems of Schwarzschild Metric}

Schwarzschild metric is

$$
\mathrm{d} s^{2}=\left(1-\frac{2 G M}{r}\right) \mathrm{d} t^{2}-\left(1-\frac{2 G M}{r}\right)^{-1} \mathrm{~d} r^{2}-r^{2}\left(\mathrm{~d} \theta^{2}+\sin ^{2} \theta \mathrm{d} \varphi^{2}\right) .
$$

Because the precession angle of Mercury orbit predicted by (47) is consistent with the observation, it is generally believed that it is correct. However, there are also some serious problems with the metric; for example, it exposes the incompatibility of electromagnetic theory and gravitational theory: when a charged particle moves in the radial direction at a speed $\mathrm{d} r / \mathrm{d} t \approx 1$, near the singularity $r=2 G M$, $\mathrm{d} s^{2} / \mathrm{d} t^{2}=(1-2 G M / r)-(1-2 G M / r)^{-1}(\mathrm{~d} r / \mathrm{d} t)^{2}<0$,

which is ridiculous because there is no reason to think that the electromagnetic equipment in the gravitational field cannot make the speed of a charged particle close to 1 .

In order to avoid the defect with (47), textbooks do not interpret $r$ in (47) as the usual radius but instead refer to it as the radial parameter with fuzzy meaning $[7,8]$. But this is unhelpful and leads only to conceptual confusion since it has been used as the usual radius when calculating the precession angle and the bending angle of light; there should be no other explanation.

Now, we calculate the ordinary pressure given by the Schwarzschild interior metric, from which we can see the defects of the Schwarzschild metric and the necessity of modifying the field equation. According to the definite of pressure, it refers to the stress per unit area. It may as well let the celestial body be a fluid with $\rho=$ const, $P_{c}$ denotes the common stress, and the common pressure given by the interior solution of Schwarzschild is

$$
\begin{aligned}
P_{c} & =\rho \int_{r}^{r_{e}} \Gamma_{00}^{1} \mathrm{~d} r=\rho \int_{r}^{r_{e}} \frac{\partial B}{A \partial r} \mathrm{~d} r=\rho \int_{r}^{r_{e}} \frac{1}{A} \mathrm{~d} B \\
& =\frac{\rho}{4} \int_{r}^{r_{e}}\left(1-\frac{8 \pi G \rho}{3} r^{2}\right) \mathrm{d}\left(3 \sqrt{1-8 \pi G \rho r_{e}^{2} / 3}-\sqrt{1-8 \pi G \rho r^{2} / 3}\right)^{2} \\
& =-\frac{3 \rho}{8}\left(1-\frac{8 \pi G \rho r_{e}^{2}}{3}\right)^{2}+\frac{\rho}{2} \sqrt{1-\frac{8 \pi G \rho r_{e}^{2}}{3}}\left(1-\frac{8 \pi G \rho r^{2}}{3}\right)^{3 / 2}-\frac{\rho}{8}\left(1-\frac{8 \pi G \rho r^{2}}{3}\right)^{2} .
\end{aligned}
$$

In weak field approximation, $P_{c}=\rho\left(G M / r_{e} n-q\right.$ $\left.G M r^{2} / r_{e}^{3}\right)$, which is Newton's result. And at the center, (48) gives

$$
P_{c}=-\frac{3 \rho}{8}\left(1-\frac{8 \pi G \rho r_{e}^{2}}{3}\right)^{2}+\frac{\rho}{2} \sqrt{1-\frac{8 \pi G \rho r_{e}^{2}}{3}}-\frac{\rho}{8}
$$

Obviously, for $1-8 \pi G \rho r_{e}^{2} / 3=0, \quad P_{c}<0$, and for $1-8 \pi G \rho r_{e}^{2} / 3=1 / 9, P_{c}=0$, which are abnormal because the pressure should not be zero anyway.
But, the common pressure given by (39) is

$$
\begin{aligned}
P_{c}=\rho \int_{r}^{r_{e}} g \mathrm{~d} r & =\rho \int_{r}^{r_{e}} \Gamma_{00}^{1} \mathrm{~d} r=\rho \int_{r}^{r_{e}} \frac{\partial B}{B \partial r} \mathrm{~d} r=\rho \int_{r}^{r_{e}} \frac{\partial B}{B \partial l} \mathrm{~d} l \\
& =\rho^{2} \int_{l}^{l_{e}} \frac{8 \pi G l}{1+4 \pi G \rho l^{2} / 3} \mathrm{~d} l=\rho \ln \frac{1+4 \pi G \rho l_{e}^{2} / 3}{1+4 \pi G \rho l^{2} / 3},
\end{aligned}
$$


in which $l=l(r)$ and $l_{e}=l\left(r_{e}\right)$ satisfy (38). There is no singularity in (50). Under weak field approximation, $l \longrightarrow r$, $P_{c}=\rho\left(G M / r_{e} n-q G M r^{2} / r_{e}^{3}\right)$, which is just the result of Newton.

\section{The Application of (46) in Cosmology}

In the comove coordinate system $(t, l, \theta, \varphi)$, the metric that describes cosmic space is the Robertson-Walker metric:

$$
\mathrm{d} s^{2}=\mathrm{d} t^{2}-R^{2}(t)\left(\frac{1}{1-k l^{2}} \mathrm{~d} l^{2}+l^{2} \mathrm{~d} \theta^{2}+l^{2} \sin ^{2} \theta \mathrm{d} \varphi^{2}\right),
$$

where $R(t)$ is the cosmic scale factor and $k$ is a constant. $l$ is the radial coordinate, and in other books it is denoted by $r$, just to distinguish it from the usual radius, here $l$ instead of $r$. When the new field equation (46) is applied to the universe, that is, combined with the Robertson-Walker metric, the following two equations are given:

$$
\begin{aligned}
\left(\frac{\mathrm{d} R}{\mathrm{~d} t}\right)^{2}+k & =-\frac{4 \pi G}{3} \rho R^{2}, \\
\frac{\mathrm{d} \rho}{\mathrm{d} t} R+3 \frac{\mathrm{d} R}{\mathrm{~d} t}(p+\rho) & =0 .
\end{aligned}
$$

Equation (52) shows that $k$ must be negative, which proves that space-time is infinite. Equation (52) is similar to the original Friedman equation; just replace $G$ there with $-(G / 2)$. Equation (53) is the so-called energy equation, which is in the same form as the original. Now putting $p=$ $-\rho$ in (52), we obtain $p=-\rho=$ const, which means that the density and pressure remain the same while the universe expands or contracts, and new matter must be created continuously in the universe. The solution of (52) is

$$
R(t)=k_{1} \sin \left(2 t \sqrt{\frac{\pi G \rho}{3}}+k_{2}\right)
$$

which shows that the universe expands and contracts in cycles. Here, $k_{1}$ and $k_{2}$ are two integral constants. Since time has no beginning and no end, the moment of $R(t)=0$ has occurred countless times. Let us define the nearest moment of $R(t)=0$ as zero; that is to say, at the moment $t=0$, then $k_{2}=0$. Hubble parameter:

$$
H(t) \equiv \frac{\mathrm{d} R}{R \mathrm{~d} t}=\sqrt{\frac{4 \pi G \rho}{3}} \cot \left(t \sqrt{\frac{4 \pi G \rho}{3}}\right) .
$$

Since everything disappears at $R(t)=0$ [17], including light, the universe in the last cycle is unobservable and no concern to us. What we care about is the age of our universe, which is the time from the beginning $(t=0)$ of the most recent cycle to today, and using (55), we obtain our universe's age:

$$
t_{0}=\frac{1}{\sqrt{4 \pi G \rho / 3}} \arctan \sqrt{\frac{4 \pi G \rho}{3 H_{0}^{2}}} .
$$

By substituting the observed density $\rho=3.1 \times 10^{-28} \mathrm{~kg} / \mathrm{m}^{3}$ of the universe and the Hubble parameter of today $H_{0}=H\left(t_{0}\right)=70 \mathrm{~km} \cdot \mathrm{s}^{-1} \cdot \mathrm{Mpc}^{-1}$ into the above equation, we get $t_{0}=1.37 \times 10^{10}$ years, that is, 13.7 billion years, the same as the previous theoretical results.

It can be seen from (54) that the cyclical period of expansion and contraction of the universe is $2 \pi / \omega=\sqrt{3 \pi / G \rho}=2 \times 10^{11}$ years, namely, 200 billion years, so the universe is currently in the expansion stage and will begin to contract in 36.3 billion years. Contraction is the reverse course of expansion.

Now, we derive the new relation between distance and redshift given by (46). Similar to the previous operation, letting the light given out by distant galaxy at the time $t$ in past and reach the Earth at the time $t_{0}$ of today, its redshift $z=\left(\lambda_{0}-\lambda_{e} / \lambda_{e}\right)=\left(R\left(t_{0}\right) / R(t)\right)-1 . \lambda$ is wavelength. We may as well put today's $R\left(t_{0}\right)=1$. Note that the subscript 0 represents today. And differentiating $1+z=1 / R(t)$, we get

$$
\mathrm{d} z=-\frac{\mathrm{d} R}{R^{2}(t)}=-\frac{\mathrm{d} R}{R \mathrm{~d} t} \frac{\mathrm{d} t}{R} .
$$

And the derivative of equation (52) gives $4 \pi G \rho / 3 H^{2}=-R \ddot{R} / 3 H^{2}$, where $H=\mathrm{d} R / R \mathrm{~d} t, \dot{R}=\mathrm{d} R / \mathrm{d} t$.

Writing today's $4 \pi G \rho / 3 H_{0}^{2}=q_{0}, H\left(t_{0}\right)=H_{0}$, and applying (52) to today, we have

$$
\begin{aligned}
k & =-H_{0}^{2}\left(1+q_{0}\right), \\
H^{2} & =-\frac{4 \pi G \rho}{3}-\frac{k}{R^{2}(t)}=H_{0}^{2}\left[\left(1+q_{0}\right)(1+z)^{2}-q_{0}\right] .
\end{aligned}
$$

On the other hand, for the motion of light,

$$
\begin{aligned}
-\frac{\mathrm{d} l}{\sqrt{\left(1-k l^{2}\right)}=\frac{\mathrm{d} t}{R(t)}} & =-\frac{\mathrm{d} z}{H} \int_{0}^{l} \frac{\mathrm{d} l}{\sqrt{1-k l^{2}}} \\
& =\frac{1}{H_{0}} \int_{0}^{Z} \frac{\mathrm{d} z}{\sqrt{\left(1+q_{0}\right)(1+z)^{2}-q_{0}}} .
\end{aligned}
$$

Note that $l$ as superscript of the integral sign refers to the galaxy's unchanged comoving coordinate. Using the relation between luminosity distance and redshift $d_{L}=(1+z) R\left(t_{0}\right) \int_{0}^{l} \mathrm{~d} l / \sqrt{1-k l^{2}}$ and completing the integration of the right of (65), we get

$$
H_{0} d_{L}=\frac{z+1}{\sqrt{q_{0}+1}} \ln \frac{(z+1) \sqrt{q_{0}+1}+\sqrt{\left(q_{0}+1\right)(z+1)^{2}-q_{0}}}{1+\sqrt{q_{0}+1}} .
$$

Here, $d_{L}$ is Luminosity distance and $q_{0}$ is the deceleration parameter today. As $z \longrightarrow 0$, expanding it, we have

$$
H_{0} d_{L}=z+\frac{1-q_{0}}{2} z^{2}+\frac{3 q_{0}^{2}-2 q_{0}-1}{6} z^{3}+\cdots,
$$

which is classical Hubble law after omitting high-order terms. The conclusion of (60) is in good agreement with the observed distance and redshift data [9-19], which strongly indicates that the modified field equation (46) is correct, the so-called dark energy does not have to exist, and the expansion of the universe is still decelerating. The curve in Figure 1 is the simulation of (60) with $q_{0}=0.14$ and 


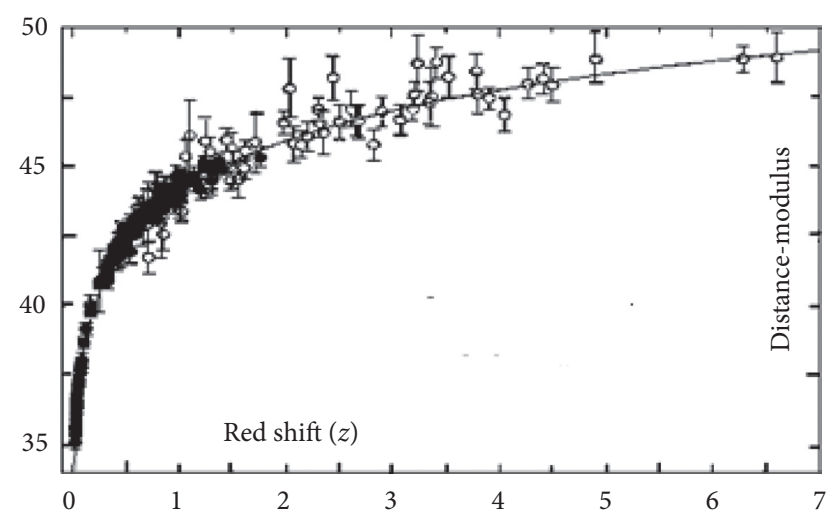

Figure 1: The recent Hubble diagram of 69 GRBs and 192 SNe Ia.

$H_{0}=70 \mathrm{~km} \cdot \mathrm{s}^{-1} \cdot \mathrm{Mpc}^{-1}, H_{0}$ is Hubble parameter of today, distance-modulus is equal to $5 \lg d_{L}+25$, and unit of $d_{L}$ is Mpc.

Note that according to the observation of $\rho$, people deduce $q_{0}=4 \pi G \rho / 3 H_{0}^{2}=0.1 \pm 0.05$.

The redshift-distance relation derived from the original field equation cannot explain the observation, in order to be consistent with the observation, dark matter and dark energy must be introduced temporarily, but such an operation has no scientific value because dark matter and dark energy are no different from the copy of ether, and in essence, they belong to the pseudoscientific concept that can never be verified by experiments. The accelerating expansion of the universe advocated by some people cannot be consistent with the facts. Though the data of the distance and redshift they measured are right, the theoretical basis for analyzing these data is wrong; that is to say, the middle derivation from data to conclusion is wrong.

\section{Galaxies and Celestial Bodies are Formed by Gradual Growth rather than by the Convergence of Existing Matter}

Since the negative pressure is confined to the inner part of the celestial body, the new matter can only generate in the celestial body not wherever. Applying $T_{v ; \mu}^{\mu}=0$ to a celestial body's interior, we obtain $\mathrm{d} m=\mathrm{d}(\rho V)=-p \mathrm{~d} V=\rho \mathrm{d} V$. Here, $V$ is the volume of the object, $m$ is its mass.

In order to keep the density of the universe unchanged during the expansion process, the celestial body must grow with time and its volume satisfies $V \propto R^{3}(t)$. From $\mathrm{d} m=\rho \mathrm{d} V=(m / V) \mathrm{d} V$, we know $m=C V$ and $C$ is integral constant, so we obtain $m \propto R^{3}(t)$; that is, for any two moments $t_{1}$ and $t_{2}$,

$$
\frac{m\left(t_{1}\right)}{m\left(t_{2}\right)}=\frac{R^{3}\left(t_{1}\right)}{R^{3}\left(t_{2}\right)}
$$

Further, $\mathrm{d} m=3 \mathrm{Hm}$. Of course, (62) is also suitable for describing the mass change of a galaxy. So, we get a new picture of the evolution of the universe: everything is expanding in Hubble; not only is the space between galaxies expanding, but the galaxies themselves are expanding, and new matter is continuously generating in galaxies. In a word, just like the night sky we see with a magnifying glass, everything is expanding but the periods of various rotations and revolutions are unchanged. The essence of cosmic expansion is the simultaneous generation of space and matter.

Many people have recognized the fractal structure of the universe, but they are unwilling to explain the formation of galaxies as the growth of fractals $[17,18]$; the obstacle is obviously that people do not know the mechanism of the generation of new matter. Now, the generation of matter is no longer a problem.

Figure 2 is a step-by-step magnification of the Solar System. It represents the actual growth process of the Solar System. With the universe expanding, the Solar System is becoming bigger and bigger; not only do its size and mass increase, but also brightness increases. For example, the Earth is moving away from the Sun at a speed of $v_{0}=H_{0} s_{0}=9 \mathrm{~m} /$ year, namely, following Hubble expansion. Since the Hubble expansion does not change the revolution period, the revolution speed of the Earth increases today at a rate of $H_{0} \bar{v}_{0}=61 \mathrm{~m} /$ year $^{2}$, and, accordingly, the mass of the Sun increases at a rate of $3 H_{0} M_{0}=4 \times 10^{20} \mathrm{~kg} /$ year. Here, $s_{0}=1.49 \times 10^{8} \mathrm{~km}$ is the distance between the sun and the Earth today, $M_{0}=2 \times 10^{30} \mathrm{~kg}$ is the mass of the Sun, and $\bar{v}_{0}=30000 \mathrm{~m} / \mathrm{s}$ is the revolution speed of the Earth today.

Again, in addition to the tide, the expansion of Hubble recedes the Moon $2.7 \mathrm{~cm}$ away from the Earth every year, the tide only recedes the Moon $1.1 \mathrm{~cm}$ away from the Earth, and, meanwhile, the radius of the Earth increases at a speed of $v=H_{0} r_{0}=0.5 \mathrm{~mm} /$ year, the Earth's mass increases at a rate of $3 H_{0} m_{0}=1.2$ billion tons per year, $r_{0}=6400 \mathrm{~km}$ is the radius of today's Earth, and $m_{0}=5.96 \times 10^{24} \mathrm{~kg}$ is the Earth's mass of today. The Earth's rotation is slowing down at a rate of $3.8 \mathrm{~cm} /$ year, just because of the tide and not the Hubble expansion. If the $3.8 \mathrm{~cm} /$ year is all the effect of tides, the result calculated according to the theory of tidal damping is that the rotation period of the Earth increases by 1.7 millisecond every year, which is inconsistent with the observation, and if the tides make the Moon only $1.1 \mathrm{~cm}$ away from the earth every year, the calculated result is that the Earth's rotation period slows down by 0.6 millisecond per year, which is in consistence with observation. Of course, these data belong to today and do not represent the past, and if you want to infer the past or future situation, you need to do a similar derivation; I will not discuss it here.

Figure 3 is a step-by-step magnification of the Milky Way. It represents the actual growth process of the Milky Way. With universe expansion, not only do its size and mass increase, but also its brightness increases. That is to say, all parts of it have been expanding according to Hubble; at the same time, new matter is continuously generated in the celestial bodies. For example, the radius of the galactic disk (refers to the luminous part) is expanding at a rate of $v_{0}=H_{0} d_{0}=600 \mathrm{~m} / \mathrm{s} ; d_{0}=30,000$ light-years is the radius of the luminous part of the galactic disk today. The Solar System is moving away from the center at a rate of $v_{0}=H_{0} r_{0}=450 \mathrm{~m} / \mathrm{s} ; r_{0}=8.5 \mathrm{kpc}$ is the distance of the Sun to the galactic center. 

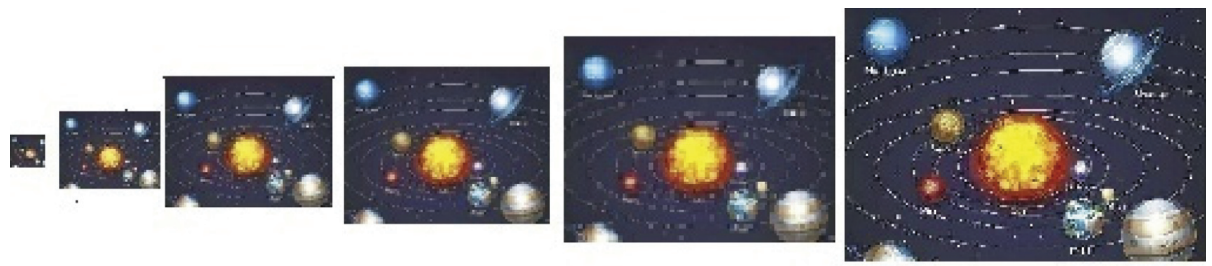

Figure 2: Schematic diagram of the growth process of the solar system with time.

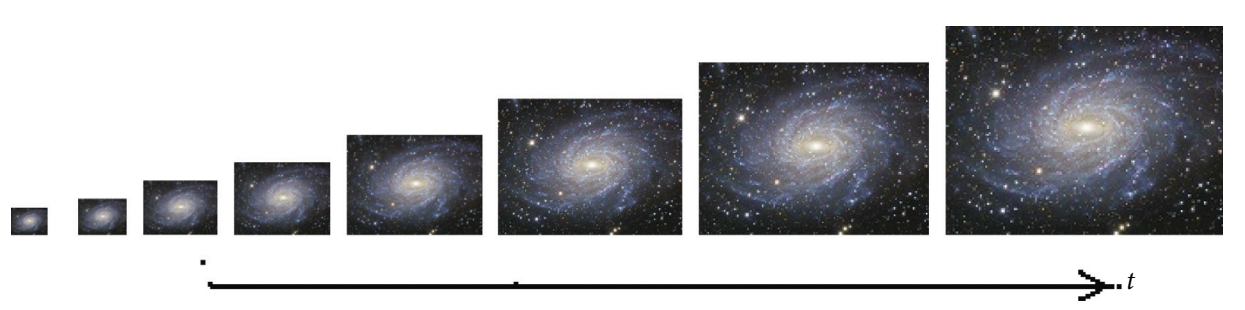

Figure 3: Schematic diagram of Gradual growth of the Milky Way.

It is because the Milky Way is formed by gradual growth, not by the accumulation of existing matter that its spiral arms are not getting tighter and tighter; otherwise, they would have been destroyed.

Figure 4 is a step-by-step magnification of a piece of cosmic space, which represents the actual expansion process of cosmic space. The white spot in the figure represents galaxies, not only is the space between galaxies expanding, but also the galaxies themselves are expanding. It tells that the more backward we look, the more evenly matter is distributed, which is just reflected by the microwave background radiation. Therefore, we say that the microwave background radiation is the comprehensive effect of redshifted photons emitted by the matter at a distant and indistinguishable distance on our instrument, and these photons have a blackbody spectrum because they come from different stars. This is a simple and realistic explanation, but it is like a myth to describe it as a relic or sound of the big bang. The distant sky we see with the naked eye is uniform, and, similarly, the distant sky we see with the telescope should be also uniform. It is shameless to deliberately tie microwave background radiation with the big bang.

It should be noted that the inverse process of the expansion of the universe is its contraction, and in the contraction process all galaxies and space atrophy reversibly.

For a more detailed discussion of the expansion process of the universe and the fractal structure of galaxies, see the author's paper and related papers [17-22].

\section{The Temperature and Brightness of Celestial Bodies Are Increasing}

It is found that the mass of a celestial body is related to its luminosity, generally speaking, the greater the mass, the greater the luminosity. For a main sequence star, we have the following empirical formula:

$$
\frac{L}{L_{\odot}}=\left(\frac{M}{M_{\odot}}\right)^{4}
$$

$L$ is the luminosity of the star and $L_{\odot}$ and $M_{\odot}$ are, respectively, luminosity and mass of the sun. The brightness and temperature of celestial bodies have the following relations:

$$
L=4 \pi r_{e}^{2} \cdot \sigma T_{e}^{4}=4 \pi r_{e}^{2} \cdot l_{e}=4 \pi d_{p}^{2} \cdot \sigma T_{p}^{4}=4 \pi d_{p}^{2} \cdot l_{p},
$$

where $l_{e}$ is absolute brightness of the star, $l_{p}$ is its vision brightness, $d_{p}$ is the distance from the star to us, $\sigma$ is Stefan-Boltzmann constant, and $T_{e}$ and $T_{p}$ are the temperature of surface and the vision temperature, respectively.

Now we treat $M$ as a variable, namely, $M \propto R^{3}(t)$. Since $r_{e} \propto R(t), d_{p} \propto R(t)$, for the same star, at any two moments $t_{1}$ and $t_{2}$, we have following relations:

$$
\frac{l_{e}\left(t_{1}\right)}{l_{e}\left(t_{2}\right)}=\frac{l_{p}\left(t_{1}\right)}{l_{p}\left(t_{2}\right)}=\frac{T_{e}^{4}\left(t_{1}\right)}{T_{e}^{4}\left(t_{2}\right)}=\frac{T_{p}^{4}\left(t_{1}\right)}{T_{p}^{4}\left(t_{2}\right)}=\frac{R^{10}\left(t_{1}\right)}{R^{10}\left(t_{2}\right)} .
$$

And assume $t_{2}=t_{0}=1.37 \times 10^{10}$ years, which is our universe age, then 1 billion years ago $t_{1}=1.27 \times 10^{10}$ years, and using (52) and the approximated formula $x \approx \sin x$ for $x \longrightarrow 0$, we have

$$
\begin{gathered}
\frac{l_{p}\left(t_{1}\right)}{l_{p}\left(t_{2}\right)}=\frac{l_{e}\left(t_{1}\right)}{l_{e}\left(t_{2}\right)}=\left(\frac{1.27}{1.37}\right)^{10}=0.46, \\
\frac{T_{p}\left(t_{1}\right)}{T_{p}\left(t_{2}\right)}=\frac{T_{e}\left(t_{1}\right)}{T_{e}\left(t_{2}\right)}=\left(\frac{1.27}{1.37}\right)^{2.5}=0.82,
\end{gathered}
$$

which means that the Sun's brightness was less than half of today's and the temperature of the solar light is $82 \%$ of today 1 billion ago. For the change of temperature of the surface of the Earth, we can also roughly estimate to use (15), if the Earth's surface temperature is $25^{\circ} \mathrm{C}(298 \mathrm{~K})$ today, 1 billion years ago the temperature was $246 \mathrm{k}\left(-27^{\circ} \mathrm{C}\right)$, and in 30 


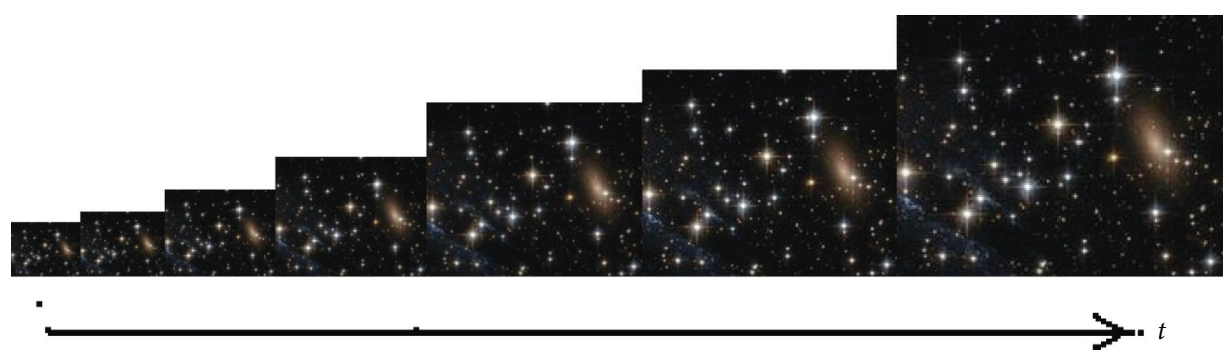

FIgURE 4: Schematic diagram of the generation process of cosmic space.

billion year its temperature will reach $6000 \mathrm{k}\left(5727^{\circ} \mathrm{C}\right)$, which is equal to the surface temperature of the Sun today. And as the universe will contract in 3.6 billion years, it can become reality for the Earth to shine like the Sun today.

Similarly, the evolution of gravity acceleration on the surface of the Earth can be deduced; 1 billion years ago the acceleration of gravity on the surface was

$$
g\left(t_{1}\right)=g\left(t_{2}\right) \frac{R\left(t_{1}\right)}{R\left(t_{2}\right)}=10 \mathrm{~m} / \mathrm{s}^{2} \times \frac{1.27}{1.37}=9.2 \mathrm{~m} / \mathrm{s}^{2} .
$$

Today's atmospheric pressure is $101 \mathrm{kPa}$, since density does not change and the height of the atmosphere increases following Hubble expansion; then, 1 billion years ago, the atmospheric pressure was

$$
P_{c}\left(t_{1}\right)=P_{c}\left(t_{2}\right) \frac{R^{2}\left(t_{1}\right)}{R^{2}\left(t_{2}\right)}=86 \mathrm{kPa}
$$

Equation (66) tells us that planets can evolve into stars; this should be the main mechanism of star formation. We usually think that the objects that do not emit light are older objects and the luminous objects are younger; this idea should be changed. The reason why a celestial body does not emit light is that its mass is not large enough, and the second reason is that the material that makes up the celestial body is too loose. The age of a celestial body refers to the time when the celestial body exists as an independent individual, not the time when the matter that makes up the celestial body exists. The chemical composition of a celestial body should be determined by its temperature, not having a direct relationship with the existence time of the celestial body. Therefore, it may not be appropriate to use the content of radioactive elements to infer the age of celestial bodies. I do not advocate talking about the concept of celestial age.

\section{More Reasonable Derivation of Orbit Precession}

In the case of weak field and low speed, the conclusion of (12) is almost the same as that of the Schwarzschild metric. As long as $r$ in (69) is replaced by $l(r)$, the orbit equation described as (12) can be obtained. Therefore, it is advisable to derive the planetary orbit equation from the well-known Schwarzschild metric and, by the way, point out the shortcoming in the previous calculation. The orbital equation described by the Schwarzschild metric is

$$
\left(\frac{\mathrm{d} u}{\mathrm{~d} \varphi}\right)^{2}=\frac{a^{2}-1}{h^{2}}+\frac{2 G M}{h^{2}} u-u^{2}+2 G M u^{3}
$$

removing the final term, which is Newton's ellipse orbit equation. Here, $u=1 / r$, and $h=r^{2} \mathrm{~d} \varphi / \mathrm{d} s=$ const and $a=$ $(1-2 G M / r) \mathrm{d} t / \mathrm{d} s=$ const are two integral constants. The derivation of (69) can be found in any textbook and I will not repeat it here. As an initial condition, we can let the perihelion on the $x$-axis; then,

$$
\int_{0}^{\varphi} \mathrm{d} \varphi=\int_{u_{1}}^{u} \frac{1}{\sqrt{\left(a^{2}-1\right) / h^{2}+2 G M u / h^{2}+2 G M u^{3}-u^{2}}} \mathrm{~d} u,
$$

where $u_{1}$ denotes the reciprocal of the perihelion distance. On the other hand, according to the theorem of factorization, we have

$$
\begin{aligned}
& \sqrt{\frac{a^{2}-1}{h^{2}}+\frac{2 G M u}{h^{2}}+2 G M u^{3}-u^{2}} \\
& =\sqrt{2 G M\left(u-\varepsilon_{1}\right)\left(u-\varepsilon_{2}\right)\left(u-\varepsilon_{3}\right)},
\end{aligned}
$$

where $\varepsilon_{1}, \varepsilon_{2}, \varepsilon_{3}$ are the three roots of the cubic equation $\left(a^{2}-1\right) / h^{2}+2 G M u / h^{2}+2 G M u^{3}-u^{2}=0$. And since $2 G M u^{2}$ is regarded as a perturbation, two of $\varepsilon_{1}, \varepsilon_{2}, \varepsilon_{3}$ must be very close to $u_{1}$ and $u_{2}$. Therefore, as an approximation, we may as well let $\varepsilon_{1}=u_{1}$ and $\varepsilon_{2}=u_{2}$, where $u_{1}$ and $u_{2}$ are the two roots of the quadratic equation $\left(a^{2}-1\right) / h^{2}+2 G M u / h^{2}-u^{2}=0$, which corresponds to the perihelion and the aphelion. Note that there must be $\mathrm{d} u / \mathrm{d} \varphi=0$ at the extreme points. And according to Veda's theorem, $\varepsilon_{3}=1 / 2 G M-u_{1}-u_{2}$; then,

$$
\begin{aligned}
& \sqrt{2 G M\left(u-\varepsilon_{1}\right)\left(u-\varepsilon_{2}\right)\left(u-\varepsilon_{3}\right)} \\
& \quad=\sqrt{-\left(u-u_{1}\right)\left(u-u_{2}\right)\left[1-2 G M\left(u+u_{1}+u_{2}\right)\right]} .
\end{aligned}
$$

Next, (70) becomes 


$$
\begin{aligned}
\varphi & =\int_{u_{1}}^{u} \frac{1+G M u+G M\left(u_{1}+u_{2}\right)}{\sqrt{-\left(u-u_{1}\right)\left(u-u_{2}\right)}} \mathrm{d} u \\
& =-\frac{G M}{2} \int_{u_{1}}^{u} \frac{d\left[-u^{2}+\left(u_{1}+u_{2}\right) u-u_{1} u_{2}\right]}{\sqrt{-u^{2}+\left(u_{1}+u_{2}\right) u-u_{1} u_{2}}}+\frac{1+3 G M\left(u_{1}+u_{2}\right)}{2} \int_{u_{1}}^{u} \frac{1}{\sqrt{-\left(u-u_{1}\right)\left(u-u_{2}\right)}} \mathrm{d} u \\
& =G M \sqrt{-\left(u-u_{1}\right)\left(u-u_{2}\right)}-\left[1+\frac{3 G M\left(u_{1}+u_{2}\right)}{2}\right] \arccos \frac{2 u-u_{1}-u_{2}}{u_{1}-u_{2}} .
\end{aligned}
$$

Obviously, for $u=u_{2}, \varphi=\pi\left[1+3 G M\left(u_{1}+u_{2}\right) / 2\right]=$ $\pi+3 \pi G^{2} M^{2} / h^{2}$, which implies that the processional angle is $\Delta \varphi=6 \pi G^{2} M^{2} / h^{2}$.

And since $u_{1}+u_{2}=2 G M / h^{2}, \quad\left(u_{1}-u_{2}\right) /\left(u_{1}+u_{2}\right)=e$, further we have

$$
\begin{aligned}
u= & \frac{u_{1}+u_{2}}{2}+\frac{u_{1}-u_{2}}{2} \\
& \times \cos \left[\frac{2 G M \sqrt{-\left(u-u_{1}\right)\left(u-u_{2}\right)}}{2+3 G M\left(u_{1}+u_{2}\right)}\right. \\
& \left.-\varphi /\left(1+\frac{3 G M\left(u_{1}+u_{2}\right)}{2}\right)\right] \\
\approx & \frac{G M}{h^{2}}\left[1+e \cos \left(1-\frac{3 G^{2} M^{2}}{h^{2}}\right) \varphi\right],
\end{aligned}
$$

whose final step takes advantage of the formula $\cos (\alpha-\beta)=$ $\cos \alpha \cos \beta+\sin \alpha \sin \beta$ and $2 G M \sqrt{-\left(u-u_{1}\right)\left(u-u_{2}\right)} \ll 1$, $1 /\left[1+3 G M\left(u_{1}+u_{2}\right) / 2\right] \approx 1-3 G M\left(u_{1}+u_{2}\right) / 2$.

It should be pointed out that the second-order approximate solution obtained by using $1 / r=u=$ $(1+e \cos \varphi) G^{2} M^{2} / h^{2}$ as the first-order approximation is wrong, that is, the following (75) is wrong:

$$
u=\frac{G M}{h^{2}}(1+e \cos \varphi)+\frac{3}{h^{4}} G^{3} M^{3} e \varphi \cdot \sin \varphi .
$$

The shortcoming of (75) is that when $\varphi=2 n \pi$, the orbital two crossover points with the $x$-axis are always invariant, so the shape of the ellipse is not guaranteed when it rotates, and the precession angle $\Delta \varphi=6 \pi G^{2} M^{2} / h^{2}$ cannot be obtained from (75) when $\varphi$ is quite big; that is to say, the transition from (75) to $u=G M h^{-2}\left[1+e \cos \left(1-3 G^{2} M^{2} h^{-2}\right) \varphi\right]$ cannot be realized. In short, using (75) to explain the precession of Mercury is not only grudging but also causing serious other problems. And again, Einstein's original calculations were also ambiguous and cannot obtain the correct processional angle according to Einstein's calculation [14].

\section{Planetary Orbit Equations of Giving Consideration to the Expansion of Space- time: The Evolution of Planetary Orbit}

Now, let us look at the orbital equation of planets in expanding space-time, which is also the equation that determines the formation and evolution of galaxies.
Our foothold is still the spherically symmetric metric field. And for a spherically symmetric metric field, no matter its source is static, oscillatory, or variable-mass, as long as the spherical symmetry is kept, the exterior solution is still the same form, namely,

$$
\mathrm{d} s^{2}=\left(1-\frac{2 G k}{\lambda}\right) \mathrm{d} t^{2}-\left(1-\frac{2 G k}{\lambda}\right)^{-1} \mathrm{~d} \lambda^{2}-\lambda^{2}\left(\mathrm{~d} \theta^{2}+\sin ^{2} \theta \mathrm{d} \varphi^{2}\right) .
$$

That is, with $t, \lambda, \theta, \varphi$ as independent coordinate variables, (76) is the solution of the vacuum field equation $R_{\mu \nu}=0$. Do not consider the meaning of $\lambda$ for the moment, and $k$ is only thought of as a constant. The proof of (76) is similar to the proof of Birkhoff law; I will not repeat here. Equation (76) offers the orbit equation of the planets:

$$
\left(\frac{\mathrm{d} u}{\mathrm{~d} \varphi}\right)^{2}=\frac{a^{2}-1}{h^{2}}+\frac{2 G k}{h^{2}} u-u^{2}+2 G k u^{3}
$$

whose derivation is the same as (69). However, here $u=1 / \lambda$ and $\quad h=\lambda^{2} \mathrm{~d} \varphi / \mathrm{d} s=$ const. $a=(1-2 G k / \lambda) \mathrm{d} t / \mathrm{d} s=$ const. Similar to (74), we have

$$
u=\frac{G k}{h^{2}}+\frac{G k}{h^{2}} e \cos \left(1-\frac{3 G^{2} k^{2}}{h^{2}}\right) \varphi .
$$

The above is the result of the coordinate system $(t, \lambda, \theta, \varphi)$, and our purpose is to solve the orbit equation in the coordinate system $(t, r, \theta, \varphi)$. To this end, we introduce the coordinate transformation. $\lambda=l / R(t)$ and meanwhile set $k=M / R^{3}(t)$, where $R(t)$ is the cosmic scale factor, and $M=$ $M(t)$ is the mass of the central celestial body and satisfies (62). And in the light of $(11), l=l(r, t)$ satisfies

$$
l=r-2 G M(t) \ln [r-2 G M(t)]-\frac{7 G M(t)}{6}+2 G M(t) \ln r_{e}(t),
$$

Of course, without considering the expansion of spacetime, all equations must go back to the previous. Now (77) is transformed into

$$
\frac{R(t)}{l(r, t)} \approx \frac{R(t)}{r}=\frac{G k}{h^{2}}+\frac{G k}{h^{2}} e \cos \left(1-\frac{3 G^{2} k^{2}}{h^{2}}\right) \varphi,
$$

which is just the orbital equation of planets and shows that while planets are moving around the center, they recede from the center in Hubble. 


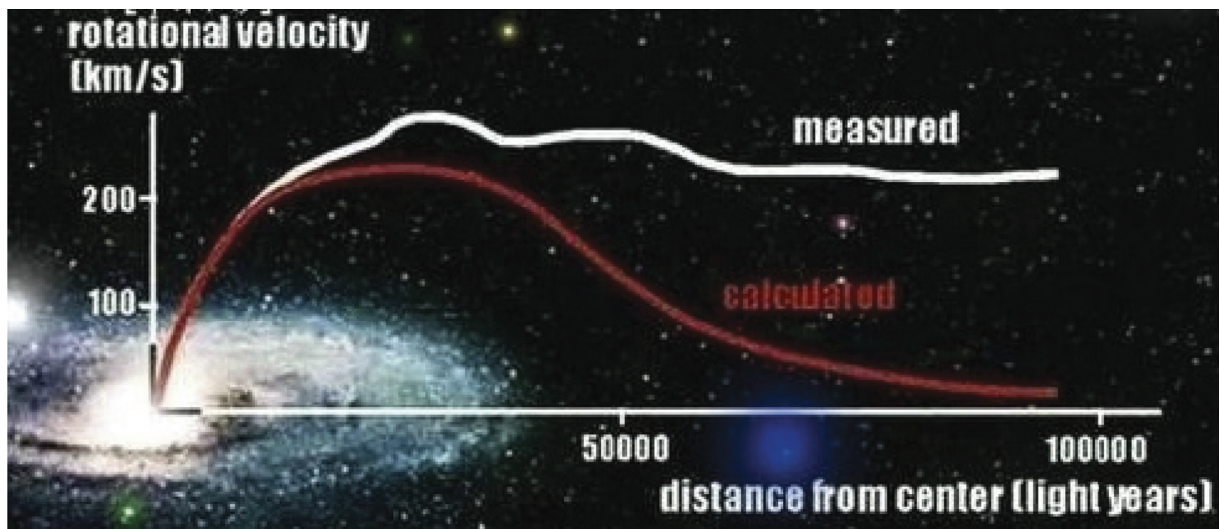

FIgURE 5: The schematic diagram of the speed distribution of matter in the Milky Way.

Besides, give consideration to Kepler's law $a^{3} / T^{2}=G M$, since $a^{3} \propto=R^{3}(t)$ and $M \propto R^{3}(t)$; then, $T=$ const. That is to say, the period of motion of planets does not change and the speed of planets increases gradually while they go away from the center.

\section{Modern Observations Do Not Confirm the Existence of Dark Matter}

Take the Milky Way as an example. It is composed of galactic ball, galactic disk, and galactic halo. Near the center, the material distribution is dense, so the galactic ball can be treated as a rotating rigid body, so that it is natural that the velocity of matter at the center is proportional to the radius, and there is no need to assume that dark matter or black hole exists. The calculated velocity of the material in the halo is lower than the measured velocity because the mass of the halo itself is ignored; that is to say, once considering the mass of the halo, there is no need to assume dark matter or black hole, too. Here is a rough estimate of the velocity of the material in the halo. Since the halo is spherical with a radius of about 100,000 light-years, at $r$ from the center, the acceleration of gravity provided by the halo itself is (let us not consider the effect of the mass of the dish):

$$
g=\frac{G M(r)}{r^{2}}=\frac{4 \pi G \rho r}{3}=\frac{v^{2}}{r},
$$

where $\rho$ is the density of the halo, $v=r \sqrt{4 \pi G \rho / 3}$ is the speed of a moving particle around the galactic center, and probably as well setting $v=30 \mathrm{~km} / \mathrm{s}$ and $r=70,000$ light-years, we obtain

$$
\rho=\frac{3 v^{2}}{4 \pi G r^{2}}=9 \times 10^{-24} \mathrm{~kg} / \mathrm{m}^{3} .
$$

That is to say, as long as the halo density reaches $9 \times 10^{-24} \mathrm{~kg} / \mathrm{m}^{3}$, the particle can maintain the speed of $30 \mathrm{~km} / \mathrm{s}$ to make a circular motion and not be thrown out of the galaxy. It is possible that the density of the halo can reach $9 \times 10^{-24} \mathrm{~kg} / \mathrm{m}^{3}$, and considering that the galactic ball and disk also have mass, even if the mass density of the halo is smaller than $9 \times 10^{-24} \mathrm{~kg} / \mathrm{m}^{3}$, the speed $30 \mathrm{~km} / \mathrm{s}$ can still be reached without being thrown out of the galaxy. Because the thickness of the galactic disk decreases slowly, its speed does not weaken, which is normal. We have no reason to deny that the density of halo can reach $9 \times 10^{-24} \mathrm{~kg} / \mathrm{m}^{3}$; such density is extremely thin. In a word, there is no need to introduce dark matter; let alone a black hole.

Figure 5 shows the speed distribution of matter in the Milky Way, the red line represents the result without considering halo mass, and the white line represents the observation result. The white line represents the observation result and is also the result of the calculation of considering the halo mass.

I do not think dark matter, dark energy, and black holes exist. Although people's observation technology is constantly improving and data is constantly accumulating, people's interpretations of the observed phenomena and data are basically wrong. The reason for this lies in the contradiction between these interpretations. Dark matter, dark energy, and black holes have pushed science into metaphysics, which is not progress but retrogression. It is shameless for those who deliberately bind the correct conclusion of general relativity with the contemporary etheric, namely, dark matter, dark energy, and black holes. It is imperative to separate general relativity from these absurd sermons. People attribute the incomprehensible phenomena to that dark matter, dark energy, and black holes are the passivation of human intelligence. In a word, all singularity physics is unreal, no matter how many halos it has.

\section{Data Availability}

The data used to support the findings of this study are available from the corresponding author upon request.

\section{Conflicts of Interest}

The author declares that there are no conflicts of interest.

\section{Acknowledgments}

The study was supported by the National Key Research and Development Plan (973 Plan), no. A030101. 


\section{References}

[1] L. Lorio, "Gravitational anomalies in the solar system," International Journal of Modern Physics D, vol. 24, no. 6, pp. 1-37, 2015.

[2] M. Ness and D. Lang, "The X-shaped bulge of the Milky way revealed bywise," The Astronomical Journal, vol. 152, no. 1, p. 14, 2016.

[3] C. Martinez-Lombilla and I. Trujillo, "Discovery of disc truncations above the galaies'smid-plane in Milky May-like galaxies," Monthly Notice of the Royal Society, vol. 483, no. 1, pp. 664-691, 2019.

[4] J. T. Nielsen, A. Guffanti, and S. Sarkar, "Marginal evidence for cosmic acceleration from type Ia supernovae," Science Reports, vol. 6, pp. 1-8, 2016.

[5] D. Herwartz, A. Pack, D. Krylov et al., "Revealing the climate of snowball Earth from $\Delta 17 \mathrm{O}$ systematics of hydrothermal rocks," in Proceedings of the National Academy of Sciences, vol. 112, no. 17, pp. 5337-5341, 2015.

[6] S. M. Som, R. Buick, J. W. Hagadorn et al., "Earth's air pressure 2.7 billion years ago constrained to less than half of modern levels," Nature Geoscience, vol. 9, no. 6, pp. 448-451, 2016.

[7] S. Weinberg, Gravitation and Cosmology, Wiley, New York, NY, USA, 2013.

[8] S. Carroll, Lecture Notes on General Relativity, Columbia University, New York, NY, USA, 2013.

[9] L. D. Landau, The Classical Theory of Fields, Pergmon Press, Oxford, UK, 1987.

[10] Einstein, The Meaning of Relativity, Princeton University Press, Princeton, NJ, USA, 1922.

[11] B. C. Tolman, Relativity, Thermodynamics and Cosmology, Oxford Clarendon Press, Oxford, UK, 1934.

[12] J. L. Yang, "Criticism to universal big bang," Astrophys and Aerospace Technology, vol. 4, p. 1, 2016.

[13] T. Felicead, "f (R) theories," Living Reviewing in Relativity, vol. 13, no. 1, p. 3, 2012.

[14] X. Mei and P. Yu, "Did LIGO really detect gravitational waves?” Journal of Modern Physics, vol. 7, pp. 1098-1104, 2016.

[15] P. Bhar and N. Pant, "Relativistic anisotropic stellar models with Tolman VII spacetime," Astrophysics and Space Science, vol. 359, no. 1, 2015.

[16] M. Yang, "Modification of gravitational field equation and rational solution to cosmological puzzles," International Journal of Physical Science, vol. 5, no. 2, 2010.

[17] J. L. Yang, "Unavoidable correction to the coupling constant in Einstein field equation," International Journal of Advanced Research in Physical Science, vol. 6, no. 11, pp. 4-30, 2019.

[18] J. Gaite, "The fractal geometry of the cosmic web and its formation," Advance in Astronomy, vol. 1, p. 25, 2019.

[19] J. de Haro, A. Paliathanasis, and R. J. Slagter, "Evolution and dynamics of a matter creation model," Monthly Notices of the Royal Astronomical Society, vol. 460, no. 2, pp. 1445-1456, 2016.

[20] S. N. Gurbatov and A. T. Saichev, "Large-scale structure of the universe," Physics-Uspekhi, vol. 55, p. 3, 2012.

[21] S. L. Blibbikov and A. D. Dolgov, "Cosmological acceleration," Physics-Uspekhi, vol. 62, no. 6.

[22] J. L. Yang, "Light speed invariant solution and its enlightenment of field equation of general relativity," Advances in Astronomy, vol. 2020, Article ID 3930947, 12 pages, 2020. 